\title{
PERFIL DE PRESCRIÇÕES DE PSICOTRÓPICOS EM FARMÁCIA COMUNITÁRIA
}

\author{
PROFILE OF PSYCHOTROPIC DRUG PRESCRIPTIONS IN A COMMUNITY PHARMACY \\ PERFIL DE PRESCRIPCIONES DE PSICOTRÓPICOS EN UNA FARMACIA COMUNITARIA \\ Andeson Gomes dos Reis \\ Maria Franciele Soares Matos \\ olindina Ferreira Melo
}

\section{RESUMO}

Este artigo tem caráter descritivo, transversal, retrospectivo e exploratório com abordagem quantitativa, respeitando os E aspectos éticos da Resolução do Conselho Nacional de Saúde (CNS) n. 466/2012. O objetivo deste estudo foi traçar o perfil de prescrições dispensadas por uma farmácia comunitária em Forquilha (CE). Os dados foram coletados a partir de notificações de receita na chamada lista "B1" de novembro de 2015 a janeiro de 2016 e novembro de 2016 a janeiro de 2017, com uso de ficha para preenchimento com as informações do prescritor, local de origem da prescrição, sexo do paciente e medicamento prescrito. Ao todo, foram coletadas 526 notificações de receita nos dois periodos de coleta e os resultados obtidos dessas notificações não variou consideravelmente quando comparados a dados de outras regiões, exceto pelo fato de que houve maior participação do especialista psiquiatra durante as prescrições. Conclui-se que é necessário traçar o perfil do usuário de psicotrópicos para melhor compreensão da realidade do município e, assim, promover o uso racional desses medicamentos.

Palavras-chave: Psicotrópico; Prescrição; Farmácia Comunitária.

1. Farmacêutico. Aluno de Especialização em Farmácia Clínica, Farmacologia e Prescrição Farmacêutica nas Faculdades Instituto Superior de Teologia Aplicada (INTA). Sobral, (CE), Brasil.

2. Aluno de graduação em Odontologia na Universidade Federal do Ceará (UFC). Sobral (CE).

3. Mestre em Bioquímica. Preceptora de Farmácia no Programa de Residência Multiprofissional em Saúde da Família da Escola de Formação em Saúde da Família Visconde de Sabóia e da Universidade Estadual Vale do Acaraú (EFSFVS/UVA). 


\section{ABSTRACT}

This article has a descriptive, cross-sectional, retrospective, and exploratory nature with a quantitative approach, complying with the ethical aspects of the Resolution of the Brazilian National Health Council (CNS) no. 466/2012. This study aimed to outline the profile of prescriptions provided by a community pharmacy in Forquilha, Ceará, Brazil. Data were collected through prescription reports in the so-called 'B1' list from November 2015 to January 2016 and from November 2016 to January 2017, by using a form to fill in the prescriber's information, place where the prescription came from, patient's sex, and medicine prescribed. Overall, 526 notification reports were collected in both collection periods and the results obtained from these notifications did not vary considerably when compared to data from other regions, except for the fact there was greater participation of the expert psychiatrist during prescriptions. We concluded there is a need to outline the psychotropic drug user's profile to a better understanding of the reality in the municipality, thus promoting the rational use of these drugs.

Keywords: Psychotropic drug; Prescription; Community Pharmacy.

\section{RESUMEN}

Este artículo tiene una naturaleza descriptiva, transversal, retrospectiva y exploratoria con un enfoque cuantitativo, que cumple con los aspectos éticos de la Resolución del Consejo Nacional de Salud de Brasil (CNS) n. ${ }^{0}$ 466/2012. Este estudio tuvo como objetivo delinear el perfil de las recetas de una farmacia comunitaria de Forquilha, Ceará, Brasil. Los datos se recogieron a través de informes de prescripciones en la llamada lista "B1" de noviembre de 2015 a enero de 2016 y de noviembre de 2016 a enero de 2017, mediante un formulario para completar la información del prescriptor, el lugar de donde provino la prescripción, el sexo del paciente y los medicamentos recetados. En general, se recogieron 526 informes de notificación en ambos períodos de recolección y los resultados obtenidos de estas notificaciones no variaron considerablemente en comparación con los datos de otras regiones, excepto por el hecho de que hubo una mayor participación del psiquiatra experto durante las prescripciones. Concluimos que es necesario delinear el perfil del usuario de psicotrópicos para una mejor comprensión de la realidad en el municipio, promoviendo así el uso racional de estos medicamentos.

Palabras clave: Psicotrópico; Prescripción; Farmacia Comunitaria.

\section{INTRODUÇÃO}

Em relação aos locais de ação dos fármacos, aqueles que agem no sistema nervoso central assumem especial importância para a humanidade, por ser mais comumente autoadministrados sem razões médicas (p. ex., anfetaminas, álcool, cafeína, nicotina) e por ter relação direta com a alteração do comportamento e a percepção da realidade do indivíduo. Tais fármacos são denominados psicotrópicos ${ }^{1}$.

Os psicotrópicos podem ser classificados quanto a estrutura química, alvo bioquímico, efeito comportamental ou uso clínico. A Portaria n. 344/1998, do Ministério da Saúde, define como psicotrópicos as substâncias que podem causar dependência física ou psíquica, relacionando tais substâncias na chamada lista "B1"2.

Os fármacos da lista "B1" são sujeitos a notificação de receita, um documento padronizado que contém informações sobre o medicamento prescrito: emitente; usuário; responsável pela compra; posologia; e quantidade de medicamento. A notificação de receita fica retida no estabelecimento responsável pelo aviamento ou dispensação do medicamento, para ser registrada no Sistema Nacional de Gerenciamento de
Produtos Controlados (SNGPC) e posteriormente encaminhada à autoridade de vigilância sanitária local².

De acordo com a segunda edição do Boletim de Farmacoepidemiologia da Agência Nacional de Vigilância Sanitária (Anvisa), de 2011, os 3 ativos de maior consumo em formulações industrializadas no Brasil por 4 anos seguidos de 2007 a 2011 foram clonazepam, bromazepam e alprazolam, nesta ordem ${ }^{3}$.

Estes três fármacos mais consumidos são psicotrópicos benzodiazepínicos com indicações para tratamento de transtorno de ansiedade, distúrbios convulsivos, tratamento adjuvante da mania aguda e outros distúrbios psiquiátricos. De modo geral, o mecanismo de ação dos benzodiazepínicos tem relação com a sensibilização dos receptores do ácido gama-aminobutírico (GABA) tipo $A$ e são intercambiáveis entre si, com as diferentes aplicações variando com características farmacocinéticas e de meia-vida ${ }^{4}$. 0 uso prolongado de benzodiazepínicos pode causar dependência e a súbita retirada do medicamento pode causar aumento dos sintomas de ansiedade, acompanhado de tremores e tontura ${ }^{5}$.

Devido ao seu potencial de abuso, é imprescindivel o estudo e a compreensão do consumo de psicotrópicos, pois 
isso gera um considerável problema de saúde pública - tanto em termos de gastos financeiros do Estado com a compra de medicamentos como de custos sociais para a população, devido aos riscos das síndromes de abstinência.

Este artigo tem por objetivo traçar o perfil de prescrições de psicotrópicos aviadas e dispensadas em uma farmácia comunitária no município de Forquilha (CE), por meio da determinação de quais substâncias são mais prescritas, qual é o sexo dos consumidores, quais são as especialidades médicas mais frequentes e quais são as principais fontes emissoras de prescrições.

\section{METODOLOGIA}

Trata-se de estudo descritivo, transversal, retrospectivo e exploratório, com abordagem quantitativa, realizado em uma farmácia comunitária em Forquilha, em um primeiro período entre novembro de 2015 e janeiro de 2016 e em um segundo período de novembro de 2016 a janeiro de 2017. 0s dados foram obtidos seguindo os seguintes aspectos: medicamento; especialidade médica; sexo do paciente; e local de origem da prescrição. Foram coletadas 325 notificações de prescrição no primeiro período (2015-2016) e 201 notificações no segundo período (2016-2017), analisadas por meio do programa computacional Libre0ffice Calc, 4.2.5.2, para quantificação das informações e posterior comparação entre os dois períodos. 0 estudo apresentou riscos mínimos, por não envolver métodos invasivos e seguir a Resolução do Conselho Nacional de Saúde (CNS) n. 466/2012, que norteia a pesquisa com seres humanos de forma direta e indireta. Obteve-se aprovação do Comitê de Ética em Pesquisa da Universidade Estadual do Vale do Acaraú, sob o Parecer n. 2.083.630, de 2017.

\section{RESULTADOS}

Ao todo, foram coletadas 526 prescrições. 0 primeiro período representou $61,78 \%$ das prescrições e o segundo período representou $38,22 \%$ das prescrições.

Nas prescrições analisadas, houve prevalência do sexo feminino $(69,85 \%$ e $73,13 \%$ no primeiro e segundo períodos, respectivamente). 0 percentual de prescrições do sexo feminino manteve-se praticamente estável nos dois períodos.

Tabela 1. Sexo observado nas prescrições coletadas nos dois períodos do estudo.

\begin{tabular}{ccccc}
\hline \multirow{2}{*}{ Sexo } & \multicolumn{2}{c}{ Primeiro periodo } & \multicolumn{2}{c}{ Segundo periodo } \\
\cline { 2 - 5 } & Frequência & Valor relativo (\%) & Frequência & Valor relativo (\%) \\
\hline Feminino & 227 & 69,85 & 747 & 54 \\
Masculino & 98 & 30,15 & 26,87 & $\mathbf{2 0 1}$ \\
\hline Total & $\mathbf{3 2 5}$ & $\mathbf{1 0 0 \%}$ & $\mathbf{1 0 0 \%}$ \\
\hline
\end{tabular}

Fonte: Elaborada pelos autores.

Como se observa a Tabela 2, a principal fonte emissora de prescrições foi a rede pública municipal (87,69\% e $84,58 \%$ no primeiro e segundo períodos, respectivamente), seguida da rede privada $(6,46 \%$ e $13,43 \%$ no primeiro e segundo períodos, respectivamente) e, por fim, da rede pública de outros municípios (5,89\% e 1,99\% no primeiro e segundo períodos, respectivamente).

Tabela 2. Origem das prescrições.

\begin{tabular}{ccccc}
\hline \multirow{2}{*}{ Origem da prescrição } & \multicolumn{2}{c}{ Primeiro periodo } & \multicolumn{2}{c}{ Segundo periodo } \\
\cline { 2 - 5 } & Frequência & Valor relativo (\%) & Frequência & Valor relativo (\%) \\
\hline Pública municipal & 285 & 87,69 & 170 & 27 \\
Privada & 21 & 6,46 & 4 & 13,43 \\
$\begin{array}{c}\text { Pública de outro } \\
\text { município }\end{array}$ & 19 & 5,89 & $\mathbf{2 0 1}$ & $\mathbf{2 0 1}$ \\
\hline Total & $\mathbf{3 2 5}$ & $\mathbf{1 0 0 \%}$ & $\mathbf{1 0 0} \%$ \\
\hline
\end{tabular}

Fonte: Elaborada pelos autores.

Os principais prescritores foram os clínicos gerais ( $72,92 \%$ e $81,59 \%$ no primeiro e segundo períodos, respectivamente), seguidos por psiquiatras $(23,69 \%$ e $10,45 \%$ no primeiro e segundo períodos, respectivamente). 0 s médicos das demais especialidades representaram 3,38\% e 7,96\% no primeiro e segundo períodos, respectivamente (Tabela 3 ). 
Tabela 3. Identificação da especialidade dos prescritores.

\begin{tabular}{ccccc}
\hline \multirow{2}{*}{$\begin{array}{c}\text { Especialização do } \\
\text { prescritor }\end{array}$} & \multicolumn{2}{c}{ Primeiro período } & \multicolumn{2}{c}{ Segundo período } \\
\cline { 2 - 5 } & Frequência & Valor relativo (\%) & Frequência & Valor relativo (\%) \\
\hline Clínico & 237 & 72,92 & 164 & 81,59 \\
Psiquiatra & 77 & 23,69 & 21 & 10,45 \\
Outros & 11 & 3,38 & 16 & 7,96 \\
\hline Total & $\mathbf{3 2 5}$ & $\mathbf{1 0 0 \%}$ & $\mathbf{3 2 5}$ & $\mathbf{1 0 0 \%}$ \\
\hline
\end{tabular}

Fonte: Elaborada pelos autores.

Como se observa na Tabela 4, os 3 medicamentos mais prescritos do primeiro período foram, em ordem, clonazepam $(66,46 \%)$, bromazepam $(12,62 \%)$ e alprazolam $(5,85 \%)$. No segundo período os medicamentos mais prescritos foram, em ordem, clonazepam $(61,69 \%)$, alprazolam $(10,95 \%)$ e bromazepam $(9,45 \%)$. A quantidade relativa de prescrições de clonazepam manteve-se praticamente estável entre os 2 períodos; houve aumento nas prescrições de alprazolam (de 5,85\% para 10,95\%). Medicamentos de tarja vermelha que não deveriam ser prescritos com notificação de receita da lista "B1" representaram apenas $1,85 \%$ das prescrições do primeiro período, mas no segundo período houve um aumento para 5,96\% das prescrições.

Tabela 4. Quantificação dos medicamentos prescritos.

\begin{tabular}{|c|c|c|c|c|c|}
\hline \multicolumn{3}{|c|}{ Primeiro periodo } & \multicolumn{3}{|c|}{ Segundo periodo } \\
\hline Medicamento & Frequência & Valor relativo (\%) & Medicamento & Frequência & Valor relativo (\%) \\
\hline Clonazepam & 216 & 66,46 & Clonazepam & 124 & 61,69 \\
\hline Bromazepam & 41 & 12,62 & Alprazolam & 22 & 10,95 \\
\hline Alprazolam & 19 & 5,85 & Bromazepam & 19 & 9,45 \\
\hline Lorazepam & 11 & 3,38 & Nitrazepam & 6 & 2,99 \\
\hline Diazepam & 11 & 3,38 & Clordiazepóxido & 4 & 1,99 \\
\hline Clordiazepóxido & 6 & 1,85 & Cloxazolam & 4 & 1,99 \\
\hline Clobazam & 6 & 1,85 & Estazolam & 3 & 1,49 \\
\hline Cloxazolam & 5 & 1,54 & Diazepam & 3 & 1,49 \\
\hline Nitrazepam & 4 & 1,23 & Clobazam & 2 & 1,00 \\
\hline Estazolam & 0 & 0 & Lorazepam & 2 & 1,00 \\
\hline $\begin{array}{c}\text { Medicamentos de } \\
\text { tarja vermelha }\end{array}$ & 6 & 1,85 & $\begin{array}{c}\text { Medicamentos de } \\
\text { tarja vermelha }\end{array}$ & 12 & 5,96 \\
\hline Total & 325 & $100 \%$ & Total & 201 & $100 \%$ \\
\hline
\end{tabular}

Fonte: Elaborada pelos autores.

\section{DISCUSSÃO}

De acordo com os 4 critérios analisados nos 2 períodos, pode-se afirmar que as prescrições são primariamente de clonazepam, para mulheres, por clínicos gerais da rede pública.

A alta frequência de prescrições para o sexo feminino nos 2 períodos $(69,85 \%$ e 73,13\%) é semelhante àquela de outras regiões do Brasil $^{6-8}$. Sugere-se que a alta prevalência de mulheres como consumidoras de medicamentos decorre de sua maior preocupação com a saúde, o que as leva a procurar os serviços de saúde com maior frequênciå ${ }^{8}$.

Comparando os dados do primeiro e do segundo período, nota-se diminuição do valor absoluto de prescrições da pública, enquanto o valor absoluto de prescrições da rede privada manteve-se praticamente estável (21 e 27). Podemse sugerir vários motivos para a diminuição de prescrições de rede municipal, como maior dificuldade na acessibilidade do medicamento; diminuição da oferta de serviço médico; dificuldade de acesso a talonários de notificação da lista B1; aumento da oferta de medicamentos na rede pública; ou menor necessidade de consumo de psicotrópicos. Independente do motivo da diminuição, a estabilidade das quantidades de prescrições indica que a rede privada aparentemente não foi afetada.

A grande quantidade de prescrições por médicos não psiquiatras também foi constatada em outros estudos, onde representavam valor acima de 50\%. Todavia, a quantidade de prescrições de psiquiatras foi consideravelmente maior do que a observada nos artigos comparados, mesmo com a diminuição do valor de $23,69 \%$ para $10,45 \%$ no segundo período do estudo ${ }^{9-12}$.

Outro estudo ${ }^{7}$ e o Boletim de Farmacoepidemiologia da Anvisa, de 2011, coincidem com os resultados que apontam 
clonazepam, alprazolam e bromazepam como os psicotrópicos mais consumidos, ao passo que há estudos ${ }^{6,10,11}$ indicando maior consumo de diazepam, seguido pelo clonazepam. As prescrições de diazepam apresentaram baixo valor relativo $(3,38 \%$ e $1,49 \%)$.

Uma pequena parcela de medicamentos de tarja vermelha foi prescrita com base nas notificações da lista B1 exclusivas para medicamentos de tarja preta, o que sugere o desconhecimento por parte dos prescritores de quais medicamentos integram essa lista.

\section{CONCLUSÃO}

0 perfil das prescrições de psicotrópicos do estudo não diferiu significativamente dos dados de outros estudos, com exceção da especialidade do prescritor, onde foi identificada a maior presença da psiquiatria em relação a outros locais.

Ainda há a necessidade de estudar o perfil dos usuários de psicotrópicos (informações como condições socioeconômicas, nivel de escolaridade e comorbidades). Evidenciou-se que a maioria dos pacientes de outro estudo não era portadora de transtorno mental que justificasse a prescrição médica, mas como há acesso mensal dos pacientes a esses profissionais, torna-se fácil exigir a prescrição de psicotrópicos junto dos medicamentos para tratar as doenças de base ${ }^{12}$.

Nosso estudo reforça a necessidade de detalhar o perfil do município em foco para promover o uso racional de medicamentos e melhorar a qualidade de vida da população.

\section{CONTRIBUIÇÃO DOS AUTORES}

Andeson Gomes dos Reis contribuiu com o delineamento da pesquisa, a coleta e análise de dados e a redação do manuscrito. Maria Franciele Soares Matos contribuiu com a redação do manuscrito. Olindina Ferreira Melo contribuiu com a redação e revisão crítica do manuscrito.

\section{REFERÊNCIAS}

1. Rang HP, Dale MM. Farmacologia. 6. ed. Rio de Janeiro: Elsevier; 2008.

2. Ministério da Saúde. Portaria n. 344, de 12 de maio de 1998. Aprova o regulamento técnico sobre substâncias e medicamentos sujeitos a controle especial [document on the internet]. Brasília (DF): Brasil; 1998 [cited 2017 May 12]. Available from: http://www.anvisa.gov.br/hotsite/ talidomida/legis/Portaria_344_98.pdf

3. Brasil. Panorama dos dados do Sistema Nacional de Gerenciamento de Produtos Controlados: um sistema para o monitoramento de medicamentos no Brasil. Brasília (DF): Agência Nacional de Vigilância Sanitária; 2011.
4. Brunton LL. As bases farmacológicas da terapêutica de Goodman \& Gilman. 12. ed. Porto Alegre: AMGH; 2012.

5. Netto MUQ, Freitas 0, Pereira LRL. Antidepressivos e benzodiazepínicos: estudo sobre o uso racional entre usuários do SUS em Ribeirão Preto-SP. Rev Ciênc Farm Básica Apl [serial on the internet]. 2012 [cited 2017 Jun 1];33(1):77-81. Available from: http://serv-bib.fcfar.unesp. br/seer/index.php/Cien Farm/article/viewFile/1777/1777

6. Lira AC. Perfil de usuários de benzodiazepínicos no contexto da atenção primária à saúde. Rev APS [serial on the internet]. 2014 [cited 2017 May 31];17(2):223-8. Available from: https://aps.ufjf.emnuvens.com.br/aps/article/ view $/ 1924 / 806$

7. Araújo FA, Lima DAS, Souza DRS. Caracterização do consumo de psicotrópicos em uma drogaria do município de santa cruz/RN. I Congresso Brasileiro de Ciências da Saúde; 15-17 Jun 2016; Campina Grande, BR. Annals.

8. Bertoldi AD, Barros AJD, Hallal PC, Lima RC. Utilização de medicamentos em adultos: prevalência e determinantes individuais. Rev Saúde Pública [serial on the internet]. 2014 [cited 2017 May 31];38(2):228-38. Available from: http:// www.scielo.br/pdf/rsp/v38n2/19783.pdf

9. Grassi LT, Castro JES. Estudo do consumo de medicamentos psicotrópicos no município de Alto Araguaia - MT. Revista Saberes [serial on the internet]. 2015 [cited 2017 Jun 2];3(1). Available from: http://www.unijipa.edu.br/media/ files/2/2_663.pdf

10. Torres MLD, Sousa LMG, Melo GC, Magalhães Júnior AA, Firmo WCA. Prescrição de psicotrópicos e especialidade médica: estudo em uma farmácia comercial no município do Maranhão. Revista Científica do ITPAC [serial on the internet]. 2014 [cited on 2017 Jun 2];7(4). Available from: http://www.itpac.br/arquivos/Revista/26/Artigo 4.pdf

11. Ferrari CKB, Brito LF, Oliveira CC, Moraes EV, Toledo OR, David FL. Falhas na prescrição e dispensação de medicamentos psicotrópicos: um problema de saúde pública. Rev Ciênc Farm Básica Apl [serial on the internet]. 2013 [cited on 2017 Jun 1];34(1):109-16. Available from: http:// serv-bib.fcfar.unesp.br/seer/index.php/Cien_Farm/article/ viewFile/2262/1370

12. Moura DCN, Pinto JR, Martins P, Pedrosa KA, Carneiro MGD. Uso abusivo de psicotrópicos pela demanda da Estratégia Saúde da Família: revisão integrativa da literatura. Sanare (Sobral, 0nline) [serial on the internet]. 2017 [cited on 2017 Nov 21];15(2):136-44. Available from: https://sanare. emnuvens.com.br/sanare/article/view/1048/594

Recebido em 13/06/2017 Aprovado em 13/11/2017

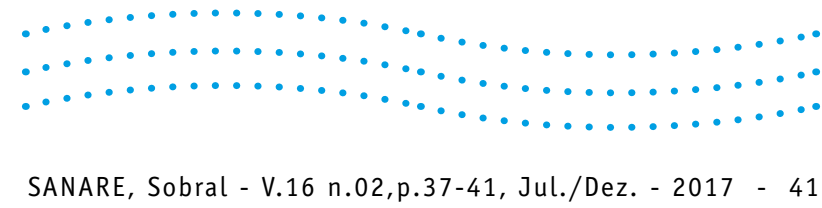

